\title{
"He's adorable and I want to take him home". Trust Perceptions Before and After First-Time Encounters with Social Robots
}

\author{
Emilia Sobolewska \\ School of Computing \\ Edinburgh Napier University \\ e.sobolewska@napier.ac.uk
}

\author{
Nicholas Vanderschantz \\ Department of Computer Science \\ University of Waikato \\ vtwoz@waikato.ac.nz
}

\author{
Francesca Bitcon \\ School of Computing \\ Edinburgh Napier University \\ 40297473@live.napier.ac.uk
}

\begin{abstract}
For users who have not interacted with a robot their perceptions are shaped by society, inculturation, and popular media. With the steady increase of robots in workplaces, during consumer encounters, and increasingly in the home, research is required to explore user perceptions of human-robot interaction and trust. This position paper aims to investigate trust between adults who have not previously interacted with a social robot, and Softbank Robotics' Nao, before, during, and after an encounter. A novel insight from our study revealed that a negative portrait of robots does not decrease trust levels.
\end{abstract}

Human-Robot Interaction, Human-Robot Trust, Social Robots, Trust Perception Score, Godspeed Questionnaire

\section{INTRODUCTION}

For many years, the depictions of robots in popular culture have been viewed as fantasies in the realm of science fiction. As technology has developed, these robots and robot-like agents are beginning to move from the pages and screens into our everyday lives. Given this, human-robot interaction studies have often considered user perceptions of trust. To date, exposure to robots in homes, workplaces, and the community is limited, and as such, acceptance and trust in robot agents may not be commonplace.

We argue that most human-robot interaction work is presently situated in the industrial workplace, or with specific sectors of vulnerable society, and therefore work is required in other sectors.

We invited adult participants to interact with a social robot for the first time and their preconceived notions about robots were analysed. Our study contributes to the growing field of human-robot trust with a specific focus on trust perceptions, before, during, and after an adult user's first-time encounters with a social robot.

\section{RELATED WORK}

As robots have been introduced into the industrial environment case studies of robot acceptance and trust in the workforce [1][2] and recommendations for the development of cobots [3] have been proposed. We seek to investigate user perception and trust of social robots which have become increasingly available commercially for home, school, and other non-industrial use.
Trust has been widely investigated in a range of contexts over many years, including interpersonal trust [4], human trust in technologies and autonomous systems [5], and human-robot trust [6]. Research has shown that with increased exposure to robots, perceived initial novelty diminishes, and preconceived ideas about robots' behaviours and capabilities can change and evolve [7]. It is considered that there are three categorisations of a person's propensity to trust a system-dispositional, situational, and learned trust [8][9]. Dispositional trust relates to a user's propensity to trust autonomous systems independent of the situation. Situational trust depends on the environment in which the system is being utilised and the users' own self-confidence in their abilities to operate it. Finally, learned trust is developed through a user's evaluations of the system with direct reference to their previous and current experience. Furthermore, research defines three types of human-technology trust [10]; functionality, helpfulness, and reliability. Functionality is the perception that the technology has the features to accomplish a specific task. Helpfulness, that technology provides adequate and responsive aid to the user. Reliability, that technology continues to operate properly and without error.

Although researchers have also looked into the association between moods and emotions and how they relate to trust [11], interestingly, to date, it has been common for human-robot studies with autonomous systems, cobots, and social robots to be undertaken at a "safe" distance with participants only observing a robot undertaking tasks [7][12]. This continued research into human-robot interaction without any direct contact has made it 
challenging to fully understand how and why trust is established. There is little evidence to show that the trust a human believes they will have when interacting with a robot will actually exist in a realworld setting.

Humanoid robots have been designed to be human-like in appearance. They usually have a head, torso, two arms, and two legs though some only have body elements from the waist up. This includes social robots pertinent to our investigations. One such humanoid robot is Nao produced by Softbank Robotics. Nao is a bipedal robot with an anthropomorphic design. It has been used in many studies with children and adults of all ages [13][14][15][16].

Anthropomorphism and anthropomorphic design are often used as interchangeable descriptors of robots though they have very different meanings. Anthropomorphic design in robots refers to the physical imitation of the human form [17] rather than the projection of characteristics onto a nonhuman agent. Anthropomorphism is the tendency of a person to attribute human qualities or behaviours to inanimate objects, particularly common with humanoid robots [18]. Engagement with social robots can be improved if they are deliberately designed to exhibit human social characteristics [17]. However, the research into the impact of the visual appearance of 2D robotic heads with three levels of human likeness revealed that participants did not find the more human-like robots in the study to be more compelling [19]. Further studies with people interacting directly with robots may help to clarify how and why anthropomorphism in interactions could influence the trust a human is willing to place in a robot.

\section{METHOD}

Our study consisted of three phases; a preintervention interview and questionnaire; a video \& audio recorded interaction with a Softbank Robotics' Nao; followed by a post-intervention interview \& a questionnaire. Interviews were audio-recorded while questionnaires were filled in by hand by the participant, and later manual transcription and analysis were completed by the researcher.

Participants were drafted using a snowball recruitment method. All of them had a working knowledge of the English language, were adults of 18 years or older, and were not part of a vulnerable group as detailed by the University Ethics requirements. Each participant met with the same researcher in a one-to-one session at the University. Overall, 9 participants (4 female/5 male) took part. The youngest participant was 18 years old and the oldest 45 years old with the mean age being 30.88 years old (SD 9.5). The majority of the participants identified as having British nationality
(6/9), with one Australian, one North American, and one Polish participant. One person completed high school education; the remaining participants have undergraduate (3/9) and postgraduate (5/9) qualifications. Five out of nine participants were currently enrolled in postgraduate computing studies at Edinburgh Napier University.

Pre-Intervention procedures included consent and demographic data collection. The participants then completed the Trust Perception Scale (TPS) [20] and Godspeed questionnaires [21] as well as the semi-structured interviews, to investigate their prior knowledge and assumptions regarding robots. We recorded a base level for human-robot trust and potential anthropomorphism the participants may experience, based on their dispositional and learned trust influences [8].

The Observation phase began with the robot having been programmed to engage with the participant by introducing itself and asking a series of questions requiring yes or no answers. Each interaction lasted approximately three minutes and was intended to give an impression of what the robot can and cannot do. The robot stood and gestured during the encounter. At the end, Nao thanked the participant for their involvement and said goodbye before sitting down and "going to sleep". Participants' facial expressions were recorded using Nao's in-built cameras. The videos were uploaded into the iMotions software to be analysed by the Affectiva facial analysis engine to investigate the situational trust [8].

Post-Intervention questionnaires and interviews investigated participants' experiences with $\mathrm{Nao}$ and whether their perceptions or assumptions regarding robots had changed. Participants were again asked to complete both the TPS and Goodspeed Questionnaires. The tools and procedures were exactly the same as for the pre-intervention phase.

\section{RESULTS}

Here we describe the results of our pre- and postintervention questionnaires and interviews as well as the emotion data captured using video footage.

The completed Trust Perception questionnaires were compiled and each participant's scores before and after their interaction with Nao were calculated [20]. Given space constraints, we report only the median TPS score before (41.67) and after (49.58) interaction for the entire participant sample. It was seen that with the exception of P5, the trust scores remained either stable (within $8 \%$ movement) or increased after the interaction with the robot. This indicates that that TPS scores did increase after interaction with Nao. 
The completed pre- and post-interaction Godspeed questionnaires were compiled and each participant's perceptions of the robot's anthropomorphism, animacy, likeability, perceived intelligence, and perceived safety was calculated [21] (Table 1).

Table 1: Godspeed Questionnaire before and after human-robot intervention

\begin{tabular}{|l|c|c|}
\hline $\begin{array}{l}\text { Semantic Differential } \\
\text { Themes (out of 5) }\end{array}$ & $\begin{array}{c}\text { Before } \\
\text { (Median) }\end{array}$ & $\begin{array}{c}\text { After } \\
\text { (Median) }\end{array}$ \\
\hline Anthropomorphism & 2 & 3 \\
\hline Animacy & 2.5 & 4 \\
\hline Likability & 3 & 5 \\
\hline Perceived Intelligence & 4 & 4 \\
\hline Perceived Safety & 4 & 4 \\
\hline
\end{tabular}

The results of the semantic differentials showed increased perceptions of Anthropomorphism, Animacy, and Likability after interaction with Nao. Perceived Intelligence and Safety were not affected by the interaction with $\mathrm{Nao}$ and remained positive.

During Pre-Human-Robot Interaction Interviews the participants were asked in broad terms, regarding their previous experiences with the robots. The results were quite uniform-four participants admitted to never having had an interaction with a robot, whilst the remaining five listed digital assistants (i.e., Apple Siri, Amazon Alexa), as their only experience. It was mentioned that irritation arises when these systems do not respond as users would expect them to. P3 described this, stating "I just want things to work. When they do not work, it's frustrating". The participants were asked whether they have familiarity with robots emerging from other sources. Some participants described watching the news, and videos from known corporations (Honda, Softbank, Boston Dynamics), robots in hospitality, care, and factories (P4, P6, P7). All of the participants were aware of the pop-cultural representation of robots in movies and literature; with a majority (5/9) pointing out, that the depiction of automatons is often negative, with two of them (P1, P6), referring to dystopian fiction.

Obviously seen lots of dystopian films. It is interesting, it is much easier to remember the bad robots than good robots, and I am just instinctively thinking of. [P1]

Only P5, P7, P9 reported no negative preconceptions of robots, based on popular culture, and recognising that their pre-interaction image of robots is manipulated and distorted.

I think some of the fictional portrayals are no doubt extremely misleading. So, things like that (...), the horror stories of the Matrix, and stuff like that. You can imagine that it gets people the heebie-jeebies, but (...) nothing that we've got at this stage is anywhere near that advanced. [P3]
When asked whether they felt robots would have a use in our schools, homes, or workplaces the answers were focused on robots being able to perform simple tasks. Two of the participants (P3, P7) were more apprehensive about the intentions of the human programmers than the robot itself.

To be honest, l'd be more concerned about the people doing the programming and their intent because with robots, being computerised systems, they essentially do what they're programmed to do... [P3]

The Observation used a combination of video recordings analysed with Affectiva Engine and researcher observations to provide cursory emotion and trust results. Due to the camera placement, both Nao's and participants' movements resulted in incomplete data for automated facial analysis. Nonetheless, Affectiva findings suggest that participants had neutral emotions for a significant portion of the interaction (mean of $70 \%$ of the time). Positive emotions were recorded on average $6.35 \%$ and negative emotions were recorded on average $0.82 \%$ of the time. While this data is of a limited sample there was very little negative emotion experienced by participants.

Instead, using video footage we manually coded observable physical or verbal cues at six key points during the study. When Nao was unveiled and stood for the first time, we observed interest $(2$ participants), amusement (4), and uncertainty (2), with one participant openly showing joy and excitement. When Nao introduced itself, six participants smiled and showed an interest, one laughed, one asked Nao to repeat itself, and one lent in to see and hear the robot. While the robot asked the first questions, we observed participants laughing and smiling (6), two participants appeared confused, while one was unengaged. When the robot made an animal noise seven participants laughed, one appeared surprised and amused, while the previously unengaged participant smiled slightly. When Nao again began asking questions, we observed our previously unengaged participant appeared to again lose interest, four participants smiled, two appeared frustrated, one thoughtful, and one participant became annoyed with Nao appearing to misunderstand. When Nao thanked participants and said goodbye most participants (5) responded with "goodbye", one with "Ok" while the remaining participants did not respond or react.

Post Human-Robot Interaction Interviews have shown, that when asked about the experience, the participants were positive, describing it as "enjoyable", "fun", "interesting" and "surprising". The robot appeared to be more interactive than they had expected. The participants have nearly unanimously expressed astonishment with the level of animation shown by Nao. Compared to the lively movements the communication was found lacking. Participants 
described problems understanding the robot and complained about needing to repeat phrases, which caused disruptions in the interaction. Several participants (P2, P5, P6, P7) expressed disappointment with the scripted nature of the interaction.

Participants anthropomorphised the robot; Nao was seen as cute in appearance; with small stature, big eyes, and adorable voice. They often referred to the robot using the pronoun 'he', P1 expressed surprise having referred to Nao in such manner: "Sometimes I find it so quiet; I wonder what he... well, that's interesting, I think 'he', not 'it' ..." [P1].

One participant mentioned the internal conflict they felt when thinking about the robot and how, for some people, this conflict could cause a barrier to interact with robots. "I could feel that instinct in my head (...) to treat it the way that I would treat a human..." [P3]. It was noted that the robot was obviously programmed to be friendly and that its' cuteness might reduce the negative media portrayals which surround robots that are too humanlike (P4). Participants P1, P2, P4 mentioned the concept of the uncanny valley, emphasising that the robot has not yet bridged that gap, which works to its benefit, allowing it to elicit more trust.

When asked if they could see this or similar technology in a social sector, responses focused on educational (P3, P5, P9) or home environments (P4, P7). P7 suggested that a robot could be used around the home/office to complete mundane tasks. It was noted that children might enjoy the simple interactions and a robot might encourage those who have difficulty engaging with traditional educational modes.

Overall, the participants found the interaction a positive experience and the robot itself an interesting, if novel, tool. P9 stating "(...) it does endear itself to you quite quickly cause it's quite pleasant and entertaining." While P6 concluded, "He's adorable and I want to take him home."

\section{DISCUSSION \& CONCLUSIONS}

With the rise of social robot development, the user acceptance by the average consumer during their personal lives raises new challenges and increases the need for investigations such as those we present here. We provide one of the few studies of humanrobot trust that involved human participants directly interacting with a social robot, whilst utilising a wide mix of quantitative and qualitative measures to capture the data. Our participants had not previously interacted with a robot and therefore we gained insight into their pre-, and post-human-robot interaction perceptions. The research revealed that although the participants' experience of robots came mostly from media, science-fiction movies, and dystopian literature, where they have damaging portrayals, it did not result in negative attitudes. Furthermore, although the participants pointed out that the child-like physical appearance of the robot was designed to be appealing and entice them, this did not reduce their levels of trust. Lastly, the recurring concern among participants is not the robot itself, but the programmer behind the code.

Given the increased trust perception by participants presented by the TPS score and the increases in perceived Anthropomorphism and Animacy presented by the Godspeed scores, further investigation of the relationship of trust and physical appearance and movement is warranted for social robots. Interestingly before interacting with the robot the Perceived Intelligence and Safety scores on the TPS questionnaire received the highest scores. While these did not increase after the interaction, they remained positive suggesting that even given negative perceptions discussed based on popculture references, participants before and after interaction were generally accepting of social robots.

The interviews conducted after the interaction with Nao have shown all participants being surprised by the robot's lively, and animated persona. The initial reactions noted during observations were positive, expressing interest, amusement, and joy, with a hint of uncertainty. All of the participants commented on the unexpected amount of movement, and life-like reactions. However, all have also discussed dissonance between human-like behaviours and underwhelming conversational skills. Participants were frustrated not experiencing social cues, and needing to repeat themselves, some interpreted the interactions as 'abrupt'. On the other hand, those negative impressions were mitigated by the overall cuteness, and childlike physical appearance of Nao, leading participants to anthropomorphize it, [17][18][19], referring to it as 'he', smiling at it, assuming intentionality in its behaviours.

Our mixed-methods approach provided a rich data set that shows promise for use with a larger sample in future work. The short interactions were enjoyable and delightful for participants, although, some traces of hesitancy and angst were detected. Longer and more complex interactions may provide both deeper understandings for the first-time users, illustrating capabilities of the social robots as well as further opportunities for investigating emotions. The rich data provided by video analysis of emotional cues appear unique to our study and, we trust, warrants further consideration. Lab setup to ensure successful capture of this, and other biometric data is required to provide new insights to the field of HRI. Finally, a more comprehensive preand post-interview may facilitate deeper analysis providing further insights into first-time human-robot interaction perceptions, vital in sustaining successful relationships with robots. 


\section{REFERENCES}

[1] Lotz, V., Himmel, S., \& Ziefle, M. (2019). You're my mate-acceptance factors for human-robot collaboration in industry. In International Conference on Competitive Manufacturing, Stellenbosch, South Africa (p. 10).

[2] Kildal, J., Tellaeche, A., Fernández, I., \& Maurtua, I. (2018). Potential users' key concerns and expectations for the adoption of cobots. Procedia CIRP, 72, 21-26.

[3] El Zaatari, S., Marei, M., Li, W., \& Usman, Z. (2019). Cobot programming for collaborative industrial tasks: an overview. Robotics and Autonomous Systems, 116, 162-180.

[4] Rotter, J. B. (1967). A new scale for the measurement of interpersonal trust 1 . Journal of personality, 35(4), 651-665.

[5] Schaefer, K. E., Chen, J. Y., Szalma, J. L., \& Hancock, P. A. (2016). A meta-analysis of factors influencing the development of trust in automation: Implications for understanding autonomy in future systems. Human factors, 58(3), 377-400.

[6] Billings, D. R., Schaefer, K. E., Chen, J. Y., \& Hancock, P. A. (2012, March). Human-robot interaction: developing trust in robots. In Proceedings of the seventh annual ACM/IEEE international conference on Human-Robot Interaction (pp. 109-110).

[7] Sanders, T., Kaplan, A., Koch, R., Schwartz, M., \& Hancock, P. A. (2019). The relationship between trust and use choice in human-robot interaction. Human factors, 61(4), 614-626.

[8] Hoff, K. A., \& Bashir, M. (2015). Trust in automation: Integrating empirical evidence on factors that influence trust. Human factors, 57(3), 407-434.

[9] Marsh, S., \& Dibben, M. R. (2003). The role of trust in information science and technology. Annual Review of Information Science and Technology, 37(1), 465- 498.

[10] Mcknight, D. H., Carter, M., Thatcher, J. B., \& Clay, P. F. (2011). Trust in a specific technology: An investigation of its components and measures. ACM Transactions on management information systems (TMIS), 2(2), 1-25.

[11]Dunn, J. R., \& Schweitzer, M. E. (2005). Feeling and believing: the influence of emotion on trust. Journal of personality and social psychology, 88(5), 736.

[12]Tussyadiah, I. P., Zach, F. J., \& Wang, J. (2020). Do travelers trust intelligent service robots?. Annals of Tourism Research, 81(C).
[13]Recio, D. L., Segura, L. M., Segura, E. M., \& Waern, A. (2013, March). The NAO models for the elderly. In 2013 8th ACM/IEEE international conference on human-robot interaction (HRI) (pp. 187-188). IEEE.

[14] Andreasson, R., Alenljung, B., Billing, E., \& Lowe, R. (2018). Affective touch in human-robot interaction: conveying emotion to the Nao robot. International Journal of Social Robotics, 10(4), 473-491.

[15]Rosi, A., Dall'Asta, M., Brighenti, F., Del Rio, D., Volta, E., Baroni, I., ... \& Scazzina, F. (2016). The use of new technologies for nutritional education in primary schools: a pilot study. Public Health, 140, 50-55.

[16] loannou, A., \& Andreva, A. (2019, September). Play and Learn with an Intelligent Robot: Enhancing the Therapy of Hearing-Impaired Children. In IFIP Conference on HumanComputer Interaction (pp. 436-452). Springer, Cham.

[17] Fong, T., Nourbakhsh, I., \& Dautenhahn, K. (2003). A survey of socially interactive robots. Robotics and autonomous systems, 42(3-4), 143-166.

[18]Fink, J. (2012, October). Anthropomorphism and human likeness in the design of robots and human-robot interaction. In International Conference on Social Robotics (pp. 199-208). Springer, Berlin, Heidelberg.

[19]Goetz, J., Kiesler, S., \& Powers, A. (2003, November). Matching robot appearance and behavior to tasks to improve human-robot cooperation. In The 12th IEEE International Workshop on Robot and Human Interactive Communication, 2003. Proceedings. ROMAN 2003. (pp. 55-60). IEEE.

[20] Schaefer, K. E. (2016). Measuring trust in human robot interactions: Development of the "trust perception scale-HRl". In Robust Intelligence and Trust in Autonomous Systems (pp. 191-218). Springer, Boston, MA.

[21]Bartneck, C., Kulić, D., Croft, E., \& Zoghbi, S. (2009). Measurement instruments for the anthropomorphism, animacy, likeability, perceived intelligence, and perceived safety of robots. International journal of social robotics, 1(1), 71-81.

[22] Sanders, T. L., MacArthur, K., Volante, W., Hancock, G., MacGillivray, T., Shugars, W., \& Hancock, P. A. (2017, September). Trust and prior experience in human-robot interaction. In Proceedings of the human factors and ergonomics society annual meeting (Vol. 61, No. 1, pp. 1809-1813). Sage CA: Los Angeles, CA: SAGE Publications 